\title{
Low-Dose and In-Painting Methods for (Near) Atomic Resolution STEM Imaging of Metal Organic Frameworks (MOFs)
}

\author{
B. L. Mehdi ${ }^{1}$, A. J. Stevens ${ }^{2}$, P. Moeck $^{3}$, A. Dohnalkova ${ }^{4}$, A. Vjunov ${ }^{1,5}$, J. L. Fulton ${ }^{1,5}$, D. M.

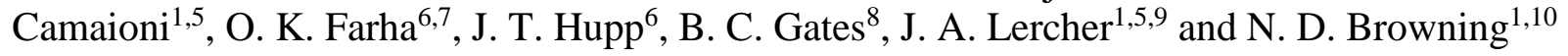 \\ 1. Physical and Computational Science Directorate, PNNL, Richland, WA, USA \\ 2. National Security Directorate, PNNL, Richland, WA, USA \\ ${ }^{3}$ Department of Physics, Portland State University, Portland, OR, USA \\ 4. Environmental Molecular Science Laboratory, PNNL, Richland, WA, USA \\ ${ }^{5 .}$ Institute for Integrated Catalysis, PNNL, P.O. Box 999, Richland, WA, USA \\ 6. Chemistry, Chemical \& Biological Engineering, Northwestern Univ., Evanston, Illinois, USA \\ 7. Chemistry, Faculty of Science, King Abdulaziz University, Jeddah, Saudi Arabia \\ ${ }^{8 .}$ Chemical Engineering, University of California-Davis, Davis, CA, USA \\ 9. Chemistry \& Catalysis Research Institute, Technische Universität München, Garching, Germany \\ 10. Materials Science and Engineering, University of Washington, Seattle, WA 98195, USA
}

Metal-organic Frameworks (MOFs) are a group of crystalline and highly porous materials consisting of inorganic metal ions/clusters (nodes) that are coordinated by organic linkers. The ability to create a wide range of porous structures, where the pore size can be easily changed in size and shape offers the potential for many applications in gas storage/separation and catalysis [1]. The presence of the organic linkers or "struts" in the sample creates challenges for high resolution microscopy as the sample itself is very sensitive to beam damage. A key challenge for understanding the structures of MOFs and how the applications can be modified by doping the nodes and changing the nature of the organic linkers, is therefore to be able to image the samples on the sub-nm length scale (the nodes are $\sim 1 \mathrm{~nm}$ ) [2].

The study of organics, where large single crystals with long-range order cannot be synthesized, is usually performed by either electron crystallography or direct imaging in the (scanning) transmission electron microscope (S/TEM). In the (S)TEM, large single crystals are not needed as the electron beam can be focused to a very small area (sub-nm if needed). The downside to this ability to see small areas is that because the electron beam has a strong interaction with the sample, it can cause significant levels of electron beam damage. However, the last 40 years of protein crystallography and more recently the use of in-situ liquid stages to study chemical reactions in the (S)TEM [3], have shown that this beam damage effect can in most cases be mitigated by the use of extremely low-dose imaging (a dose rate of less than 0.1 electrons/angstrom ${ }^{2} / \mathrm{s}$ and a cumulative dose of less than 10 electrons/angstrom ${ }^{2}$ ). In addition to simply lowering the dose through conventional means (changing the emission current and probe dwell time), more recent use of compressive sensing/in-painting methods for STEM has also been shown to lower the effective dose and dose rate [4].

Figure 1 shows a Z-contrast image obtained from an NU-1000 MOF [2] obtained from a probe corrected FEI $80-300 \mathrm{kV}$ Titan operating in the low-dose mode described above. The sample can clearly survive under these conditions long enough to obtain a full scan with a probe size of $\sim 0.1 \mathrm{~nm}$. Results obtained from doped NU-1000 MOF structures (not shown) indicate that the atomic structure of the nodes themselves can be resolved under certain imaging conditions (including the use of in-painting methods). In this presentation the results from these samples will be described in more detail, along with the experimental strategy to obtain higher resolution images in the future [5] [6]. 
References:

[1] A. J. Howarth et al, Chem. Mater. acs.chemmater. 6b02626 (2016)

[2] H. C. Zhou, J. R. Long, O. M. Yaghi, Chem. Rev. 112, (2012) p. 673.

[3] B. L. Mehdi et al, Nano Letters 15, (2015), p. 2168.

[4] A. Stevens et al, Microscopy 63, (2014), p. 41.

[5] L. Kovarik, et al, Applied Physics Letters 109, (2016), p. 164102.

[6] This work supported in part by the Chemical Imaging Initiative (CII) and the Analytics in Motion (AIM) Initiative, Laboratory Directed Research and Development (LDRD) Programs at Pacific Northwest National Laboratory (PNNL). PNNL is a multi-program national laboratory operated by Battelle for the U.S. Department of Energy (DOE) under Contract DE-AC05-76RL01830. A portion of the research was performed using the Environmental Molecular Sciences Laboratory (EMSL), a national scientific user facility sponsored by the Department of Energy's Office of Biological and Environmental Research and located at PNNL. The sample were provided by the Inorganometallic Catalysis Design Center, an Energy Frontier Research Center funded by the U.S. Department of Energy, Office of Science, Basic Energy Sciences under Award No. DE-SC0012702.

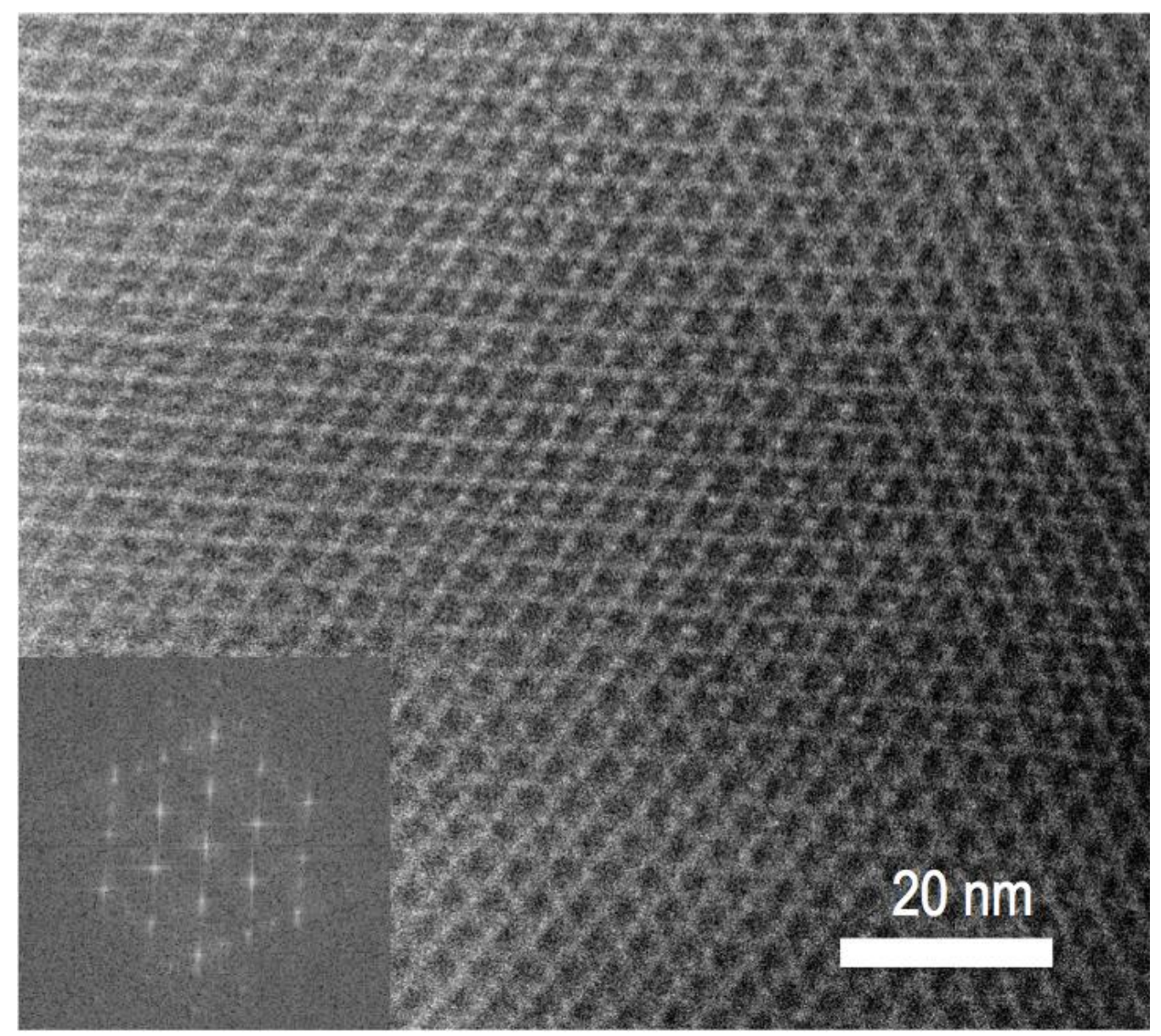

Figure 1. A high resolution Z-contrast STEM image of the NU1000 MOF in the [001] direction showing the distribution of the $\mathrm{Zr}_{6}(\mathrm{O})_{8}$ nodes (the FFT is inset). 